\title{
Application of antioxidant and ultraviolet absorber into HDPE: Enhanced resistance to UV irradiation
}

https://doi.org/10.1515/epoly-2019-0053

Received April 26, 2019; accepted July 11, 2019.

\begin{abstract}
Herein, high density polyethylene (HDPE) matrix was applied as the polymer matrix for its excellent overall performance and low price. In the study, antioxidants $(0.5 \mathrm{phr})$ and ultraviolet absorber (1 phr) were added into the polymer matrix (100 phr) to investigate their influence on UV resistance, respectively. The macroscopic properties and microstructure changes of the samples were both investigated through the characterizations of Fourier transform infrared spectroscopy (FTIR), differential scanning calorimetry (DSC), mechanical properties and thermogravimetric analysis (TGA). This work also conceived the three methods of capturing free radicals generated by photoaging, decomposing peroxides generated by photoaging, and absorbing ultraviolet light to achieve anti-aging effects. The results showed that the UV absorber improves the UV resistance of the material better than the antioxidant. After irradiation for $600 \mathrm{~h}$ under the aging condition of $0.51 \mathrm{w} / \mathrm{m}^{2} @ \lambda=340 \mathrm{~nm}$, the samples added with UV absorber also still maintain their mechanical properties at a high level. For comparison, the samples added with antioxidants presents poor mechanical properties only after irradiation for $200 \mathrm{~h}$. Besides, the crystallinity of HDPE and HDPE added with antioxidant significantly increased after irradiation, but the crystallinity of HDPE introduced with UV absorber presents slight changes before and after irradiation. Meanwhile, all the irradiated samples also maintained the original excellent
\end{abstract}

\footnotetext{
* Corresponding author: Jun Zhang, College of Materials Science and Engineering, Nanjing Tech University, Nanjing 210009, China, email: zhangjun@njtech.edu.cn.

Tiankai Jiang, College of Materials Science and Engineering, Nanjing Tech University, Nanjing 210009, China; School of International Education, Jiangsu Vocational Institute of Commerce, Nanjing 211168, China.

Yanli Qi and Yuchen Wu, College of Materials Science and Engineering, Nanjing Tech University, Nanjing 210009, China.
}

thermal stability. According to the obtained results, the aging mechanism was also analyzed.

Keywords: polyolefins; antioxidant; UV absorber; crystallization; UV resistance

\section{Introduction}

High density polyethylene (HDPE) exhibits excellent performance like low cost, dielectric and mechanical properties (1-5). Therefore, HDPE has been used in many applications such as industry, agriculture, construction and daily necessities (6-10). However, HDPE is easy to degradation and eventually aging phenomenon happens under the exposure of ultraviolet (UV) radiation, high temperature and contact with oxygen (11). The aging phenomenon finally results in a terrible shortening of service life, as well as some serious environmental problems of recycled plastics $(12,13)$. Therefore, it is of great importance to investigate the methods of antiultraviolet to extend their service life, which in turns effectively reduces energy consumption and protects the environment at the same time.

Two commercially functional particles, namely antioxidants and UV absorbers, have been widely applied to improve the UV aging resistance of HDPE. As the literature reported, antioxidants and UV absorbers both exhibit excellent influence on UV aging resistant performance (14-16). Antioxidant terminates chain reactions by trapping free radicals or decreases chain reaction rate by decomposing peroxides, improving the anti-aging property of polymer. With regard to UV absorber, it reduces the source of the molecular chain reaction by absorbing a large amount of UV light, leading to the enhancement in anti-aging property of the polymer. It is well known that the UV aging of HDPE is also affected by any parameters including catalyst traces and additives (17,18). Recently, many researchers have been focusing on the application of antioxidants and UV 
absorbers for the anti-aging purpose. J. S. Park studied the effects of antioxidant on carbon black/polyethylene conductive composites (19), meanwhile Y. Javadi studied the effect of UV absorber on the UV stability of HDPE (20). Their research is worthy of recognition, but the effects of antioxidants and UV absorbers on HDPE have not been compared and further analyzed.

Since different kinds of antioxidants, as well as UV absorbers, actually present different efficiency in UV aging resistance, it is of great significance to investigate the UV aging resistance of different kinds of antioxidants and UV absorbers. Herein, two different kinds of antioxidants, like phenolic antioxidant and phospholipid antioxidant, were utilized to investigate their efficiency in UV aging resistance. Simultaneously, benzotriazole UV absorber and benzophenone UV absorber were also applied in this work. The anti-aging effect of antioxidants or UV absorbers on HDPE was investigated. Also, their UV aging resistant performance was compared, which provides reasonable reference for the outdoor application of HDPE. The UV aging resistance was characterized by the ultraviolet accelerated aging chamber, followed by the characterizations of mechanical properties, Fourier transform infrared spectroscopy (FTIR), different scanning calorimetry (DSC) and thermogravimetric analysis (TGA).

\section{Experimental}

\subsection{Materials}

High density polyethylene (HDPE4902, density $0.95 \mathrm{~g} / \mathrm{cm}^{3}$, melting index at the temperature of $190^{\circ} \mathrm{C}$ and the load of $5 \mathrm{~kg}$ is $0.225 \mathrm{~g} / 10 \mathrm{~min}$ ) was provided by Yangzi Petrochemical Company Limited, China. Diphenylketone ultraviolet absorber (UV531, 2-hydroxy-4-n-octoxybenzophenone) was supplied by BASF, Germany. Benzotriazole ultraviolet absorber (UV326, 2-(3-tert-Butyl-2-hydroxy5-methylphenyl)-5-chloro-2H-benzotriazole) was obtained by BASF, Germany. Blocked phenol antioxidant (Irganox1010, Pentaerythritol tetrakys 3-(3,5-ditert-butyl4-hydroxyphenyl) propionate) was purchased from BASF, Germany. Phosphite antioxidant (Irgafos168, Tns-(2,4-ditert-butyl)-phosphite) was supplied by BASF, Germany.

\subsection{Sample preparation and UV exposure}

The samples were fabricated by utterly blending with ultraviolet absorbers and antioxidants using a two roll mixing mill (Shanghai Rubber Machinery Works, China). The compounds were compressed in the curing press to manufacture the sheets with the thickness of $1 \mathrm{~mm}$. During the fabricating process, the temperature was $160^{\circ} \mathrm{C}$ with the pressure of $10 \mathrm{MPa}$ for $15 \mathrm{~min}$. After that, the compounds were put into another curing press for cooling modeling under the pressure of $10 \mathrm{MPa}$. All samples were cut into dumbbell and round sheets for testing and a vernier caliper was used to measure the thickness and width. The thin neck portion of the dumbbell sheet was measured 5 times at different locations, and then the average values were applied. The artificial accelerated UV irradiation was conducted in a Xenon lamp test chamber (Q-SUN1000, Q-Lab, USA), and the wavelength range $(\lambda)$ is from $290 \mathrm{~nm}$ to $800 \mathrm{~nm}$. The standard of irradiation followed the international standard (ISO 4892-2:2006). The irradiation intensity was set at $0.51 \mathrm{w} / \mathrm{m}^{2} @ \lambda=340 \mathrm{~nm}$ and the panel temperature was maintained at $65^{\circ} \mathrm{C}$. The content of $0.5 \mathrm{phr}$ of antioxidants (Irganox1010 or Irgafos168) were added into the polymer matrix, and the corresponding samples were named as PEA1010 and PEA168, respectively. Simultaneously, the content of $1 \mathrm{phr}$ of UV absorbers (UV531 or UV326) was incorporated with the polymer matrix. The relative samples were named as PEU531 and PEU326, respectively.

\subsection{Characterizations}

Universal testing machine was used to evaluate the mechanical properties of different samples. According to GB/T1040-2006, the samples with thickness of $1 \mathrm{~mm}$ were cut into dumbbell test strips to test tensile properties at the temperature of $20^{\circ} \mathrm{C}$, including tensile strength and elongation at break. During the measurement, the samples were vertically fixed on a universal testing machine (CMT 5254, Shenzhen SANS Testing Machine Company Limited, China) machine with a speed of $50 \mathrm{~mm} / \mathrm{min}$. For each sample, five measurements were conducted and the average value was applied as the final value.

FTIR analysis was conducted with the assistance of a FTIR spectrophotometer (Nicolet IS5, Thermo Fisher, USA). The spectra were collected in the range from 600 to $4000 \mathrm{~cm}^{-1}$ with a resolution of $4 \mathrm{~cm}^{-1}$, in the reflection mode. For the fact that the chemical structures of materials easily change under the exposure of UV irradiation, carbonyl index (CI) was applied to evaluate the chemical structure changes for better understanding. $\mathrm{CI}$ is calculated according to the following equation: 


$$
C I=A_{1731} / A_{1461 \& 1472}
$$

where $A_{1731}$ is the absorbance area of carbonyl group which occurs in the irradiation process at $1731 \mathrm{~cm}^{-1}$, and $A_{1461 \& 1472}$ stands for the absorbance area of interval standard peak at 1461 and $1472 \mathrm{~cm}^{-1}$. The internal standard peak was utilized to evaluate the variation of carbonyl in process, as a result of that the structure of methyl is unaltered during the irradiation.

The thermal behaviors of samples were analyzed by DSC (DSC Q20, TA Company, USA). During the experiment, $10 \mathrm{mg}$ of samples were used for testing. Then, the testing system was heated from $40^{\circ} \mathrm{C}$ to $160^{\circ} \mathrm{C}$ under a heating rate of $10^{\circ} \mathrm{C} / \mathrm{min}$ to erase the heat history. After that, the samples were cooled to $40^{\circ} \mathrm{C}$ at a speed of $5^{\circ} \mathrm{C} / \mathrm{min}$. Eventually, the samples were heated to $160^{\circ} \mathrm{C}$ through a heating speed of $10^{\circ} \mathrm{C} / \mathrm{min}$ to obtain the melting curve. The whole process was carried out in dry nitrogen atmosphere, and the flow is $50 \mathrm{~mL} / \mathrm{min}$. The crystallinity $\left(X_{c}\right)$ can be measured by the following equation:

$$
X_{c}=\Delta H_{m} / \Delta H_{m}^{\star} \times 100 \%
$$

where $\Delta H_{m}$ is the melting enthalpy of the test in the process, and $\Delta H_{m}^{*}=277.1 \mathrm{~J} / \mathrm{g}$ is the melting enthalpy of HDPE of $100 \%$ crystallinity (21).

A thermogravimetric instrument (Q20, TA instruments, America, USA) was used to measure the loss weight of the samples. The sample for each test was about $10 \mathrm{mg}$ and the test was under the atmosphere of pure nitrogen (the flow was $40 \mathrm{~mL} / \mathrm{min}$ and lasted for $60 \mathrm{~min}$ ). Subsequently, the heating rate was $20^{\circ} \mathrm{C} / \mathrm{min}$ from $40^{\circ} \mathrm{C}$ to $700^{\circ} \mathrm{C}$.
The main use of optical microscope (NP-800TRF, Nanjing Jiangnan Novel Optics Co., Ltd. China) in this study was to analyze the morphology of the surface before and after aging of the samples.

\section{Results and discussion}

\subsection{Mechanical properties}

Figure 1 shows the mechanical properties of the samples. Each sample was tested 5 times at different aging time points. The average values were used as the final results, and the corresponding standard deviation was also included. Without UV irradiation, PEA performs higher value of the tensile strength than HDPE, and PEU as well. The increased values can be ascribed to the fact that antioxidants effectively interrupt the break of molecular chains, which results from the melting method during the manufacturing process. With the increasing time of UV irradiation, a sharp decrease can be observed with regard to the tensile strength of neat HDPE and PEA. After UV irradiation for $400 \mathrm{~h}$, the tensile strength of neat HDPE reduces from 26.7 MPa to 8.8 $\mathrm{MPa}$, while that of the PEA1010 declines from $34.3 \mathrm{MPa}$ to $16.3 \mathrm{MPa}$. At the same time, the tensile strength of PEA168 decreases from 36.7 $\mathrm{MPa}$ to $23.8 \mathrm{MPa}$. However, the tensile strength of PEU declines slightly as irradiation time increased. After irradiation for $800 \mathrm{~h}$, tensile strength of PEU531 and PEU326 still maintain at the value of around $26 \mathrm{MPa}$ and 28.2 $\mathrm{MPa}$, respectively. With regard to the elongation at break, all the samples exhibit the high value of around $700 \%$ without UV irradiation. Yet, sharp decrease can be observed on the samples of HDPE and PEA as irradiation
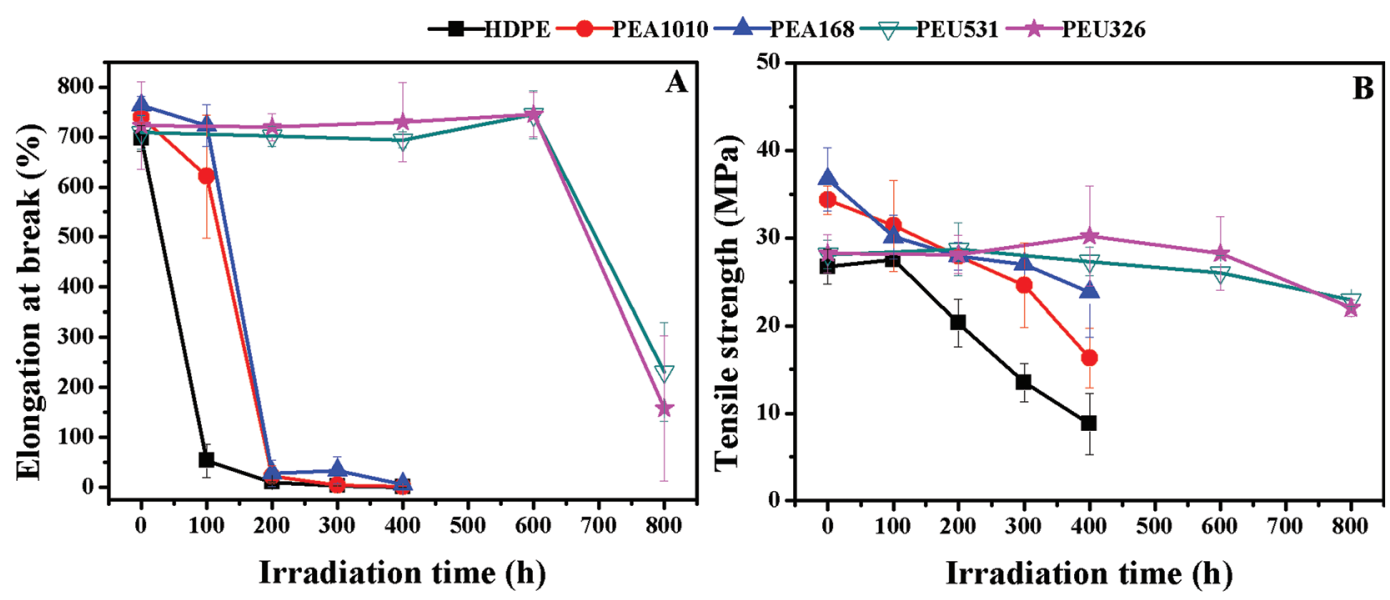

Figure 1: Elongation at break (a) and tensile strength (b) of samples under different UV irradiation time. 
time increases, where the values declines to the value lower than 50\% with UV irradiation time up to $200 \mathrm{~h}$. Simultaneously, elongation at break decreases to nearly $0 \%$ with the UV irradiation time of $400 \mathrm{~h}$. As observed, the mechanical properties of HDPE, PEA1010 and PEA168 decreased greatly after irradiated for $400 \mathrm{~h}$. The sample was too brittle and easily break, losing their values. Thus, further irradiation time up to $400 \mathrm{~h}$ is not required. However, elongation at break of PEU still maintains the value higher than $700 \%$ after irradiating for $600 \mathrm{~h}$, yet it sharply reduced after being irradiated for $800 \mathrm{~h}$. Though the elongation at break of PEU largely decreases with the UV irradiation up to $800 \mathrm{~h}$, it performs much better antiaging features than neat HDPE and PEA under the same UV irradiation time.

Based on mechanical analysis, antioxidants play little effect on the enhancement in the UV resistance of matrix, while UV absorbers present a significant improvement in the relative UV resistance property. The difference is attributed to thoroughly different mechanism effect of UV resistance, and the related mechanism is further discussed based on FTIR analysis.

\subsection{FTIR analysis}

Infrared spectrometry is a useful tool for studying the degradation of polymers $(22,23)$. Figure 2 shows the evolution of the infrared spectra of samples with different UV irradiation time. The absorptions at $2914 \mathrm{~cm}^{-1}$ and $2846 \mathrm{~cm}^{-1}$ are assigned to the asymmetrical and symmetrical stretching vibration of $\mathrm{C}-\mathrm{H}$ bond of $-\mathrm{CH}_{2}-$ groups, respectively. The peak appearing at $1472 \mathrm{~cm}^{-1}$ is ascribed to the scissoring vibration of $-\mathrm{CH}_{2}-$ groups, while the asymmetric bending vibration of $-\mathrm{CH}_{3}$ groups is observed at $1461 \mathrm{~cm}^{-1}$. The peaks occurring at $730 \mathrm{~cm}^{-1}$ and $718 \mathrm{~cm}^{-1}$ are associated with the inner rocking vibration of $-\mathrm{CH}_{2}-$ in the crystalline part and inner rocking vibration of $-\mathrm{CH}_{2}-$ in the amorphous part respectively. The peak of ester carbonyl stretching vibration closes to that of carbonyl groups. Simultaneously, the peaks at $1264 \mathrm{~cm}^{-1}$ and $1187 \mathrm{~cm}^{-1}$ are the vibration of $\mathrm{C}-\mathrm{O}$ bonds. The carbonyl stretching vibration appears at $1731 \mathrm{~cm}^{-1}$. Since the peak area of the carbonyl groups largely changes with the increasing irradiation time, the evolution of absorption intensity of the carbonyl groups is chosen to study the degradation of the samples for the obvious changes.

The carbonyl index is applied to conduct the further FTIR analysis, which is defined as the ratio of the peak area at $1731 \mathrm{~cm}^{-1}$ to that of the interval standard peak at 1461 and $1472 \mathrm{~cm}^{-1}$. Figure 3 demonstrates the changes of the CI values as a function of the irradiation time for the samples until the embrittlement of the samples hinders the achievement of reliable FTIR spectra. It can be seen from Figure 3 that unirradiated samples exhibit a small amount of carbonyl groups, which may be ascribe to the heat, oxygen and mechanical forces of the polyethylene during processing. Clearly, the CI of neat HDPE and PEA1010 sharply increases with the irradiation time of 200 h, implying easy oxidation of HDPE and PEA1010.
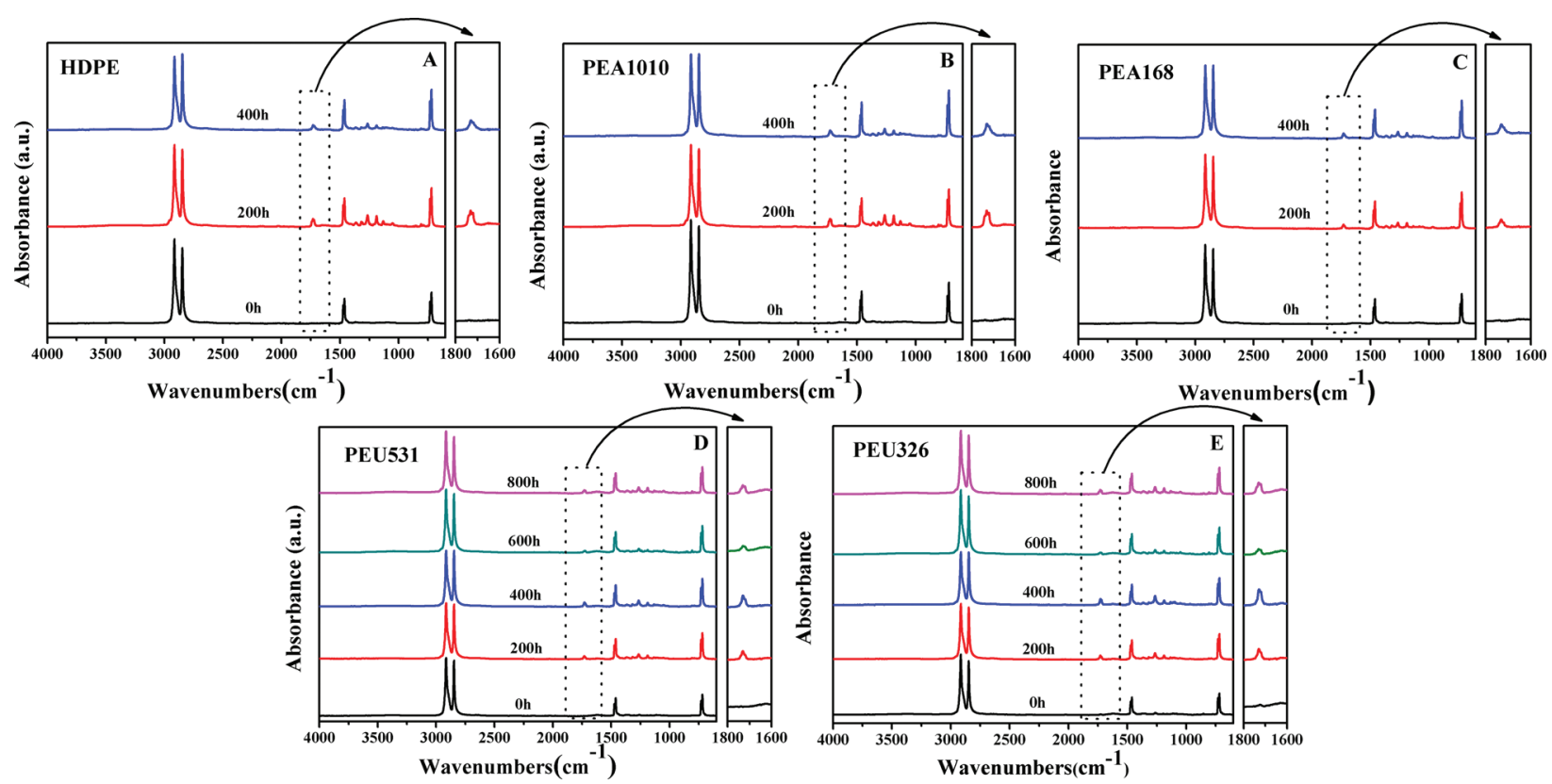

Figure 2: FTIR spectra of samples after being irradiated for different time: (a) HDPE; (b) PEA1010; (c) PEA168; (d) PEU531; (e) PEU326. 


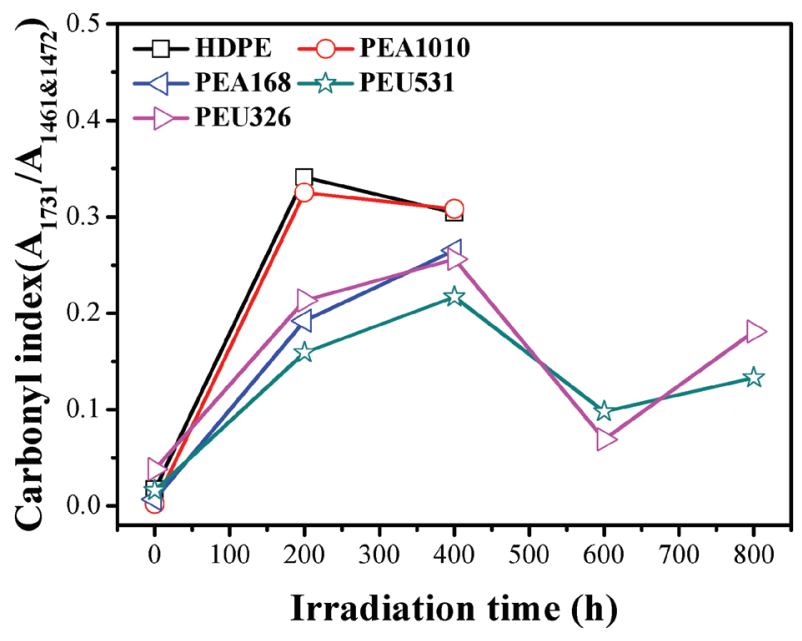

Figure 3: Carbonyl index of samples irradiated after various time.

After UV irradiation for $400 \mathrm{~h}$, the CI shows a slight decline. With regard to the PEA168, applying Irgafos168 as antioxidant, the CI exhibits a value of 0.192 after being irradiated for $200 \mathrm{~h}$. This indicates that Irganox168 inhibits carbonyl production. The CI of PEA168 continues to rise slowly until irradiation for $400 \mathrm{~h}$. Besides, the CI of PEU series become higher as the aging time increases until UV irradiation time up to $400 \mathrm{~h}$. However, after irradiation for $600 \mathrm{~h}$, a slight decline appears in the CI of PEU series. Similar results had been reported, carbonyl groups formed and were consumed to form macromolecular radicals that further combine to form new double bonds during the UV irradiation process of polyolefins (24). During the UV irradiation, the crosslinking structures are produced, which is the reason for the fact that the CI of HDPE, PEA1010, PEU531 and PEU326 decreases $(25,26)$. The carbonyl group is interrupted by high-energy ultraviolet light to form free radicals during the degradation process, and the molecular chains with free radicals react with each other to form a new crosslinking structure. After UV irradiation for $600 \mathrm{~h}$, the CI enhances with the increase of UV irradiation time. This indicates that photo-oxidative aging and cross-linking between molecular chains are a pair of competitive reactions in the UV aging process.

Molecular chain cleavage and ester group formation happen in the neat HDPE resin after prolonged aging. The photo-oxidative degradation of polymer chains is initiated by UV light. The amount of UV light absorbed by the matrix exhibits an important factor affecting photo-oxidative degradation. The more UV light is absorbed by the matrix, the more free radicals can be produced. In the initial stage, the hydrogen atoms in the macromolecular chain are taken away to form alkyl radicals. Then, the unsaturated carbon-hydrogen bonds are initiated in the photo-oxidative process to produce alkyl hydroperoxides, and this became the main process for photo-oxidative degradation. Specially, alkyl hydroperoxide radicals are the key point to the formation of ester carbonyl groups, but in turns the appearance of carbonyl groups further accelerates the aging degradation of HDPE (26). Phenolic antioxidant has active hydrogen atoms $(\mathrm{O}-\mathrm{H})$ in molecules, and this kind of hydrogen atoms is more active than that of the carbon chains (including double bonds). They can be separated to combine with the macromolecule radicals like $\mathrm{R} \cdot$ and $\mathrm{R}-\mathrm{O}-\mathrm{O} \cdot$ to form hydrogen peroxide and stable phenoxy radical (ArO.). Due to the increase of phenoxy radicals' adjacent substituent and branch, more space blocking effect can be obtained. In this case, phenoxy radicals are under the protection of bigger groups, which therefore improves their stability. In addition, phenoxy radicals and benzene rings are both in the same conjugated system. Hence, the phenoxy radicals are stable with lower activity and cannot trigger chain reaction. One phenoxy only combines with one active radical and suspends it. Then, the stable compound with end of chain reactions can be obtained. The stability of phenoxy radicals can both avoid quick consumption of antioxidants. Subsequently, less chain transfer reactions occur, leading to the improvement in oxidation resistance. Phosphate antioxidant decomposes macromolecule hydrogen peroxide $\mathrm{ROOH}$ and turns it into stable compounds to resist chain reactions. The antioxidant 1010 is a radical scavenger, which can be selectively and quickly consumed by the large amount of alkyl radicals, and low-effectively reduce the formation of ester carbonyl groups. Antioxidant 168 could decompose the macromolecular hydrogen peroxide, which can effectively reduce the formation of the ester carbonyl groups. But it cannot prevent the generation of a large number of alkyl radicals, and the improvement of the UV resistance is also limited. However, with the addition of the UV absorber, it is a kind of compound that could strongly and selectively absorb ultraviolet light, and it efficiently transforms light into heat energy by its molecular structure. Subsequently, the absorbed energy is emitted through heat energy and low-energy radiation, and therefore preventing oxidation of the samples. Since most of the ultraviolet light is absorbed and only a small amount of ultraviolet light enters the matrix, there was almost no breakage of the molecular chains even after UV irradiation for $400 \mathrm{~h}$. Also, only a small amount of ester groups can be formed, which greatly improves the $\mathrm{UV}$ resistance of the matrix. 


\subsection{DSC analysis}

The DSC was used to evaluate melting and crystallization behavior. As shown in Figure 4a, the first melting trace of each sample was obtained in this work. All DSC scans were normalized by the mass of the sample, which means that the area of the endothermic peak in the curve could roughly determines the degree of crystallinity. Only one endothermic peak can be observed in each spectrum. Higher melting peaks of HDPE and PEA1010 after UV irradiation are achieved than those peaks without irradiation, which indicates the higher absorbed enthalpy. This means that HDPE and PEA1010 exhibit higher crystallinity after UV irradiation. As can be seen from Figure 4c, the crystallinity of HDPE increases from $64.2 \%$ to $74.9 \%$, and that of PEA1010 increases from $62.2 \%$ to $74.5 \%$. Also, significant enhancement in the crystallinity of PEA168 is also obtained. However, there is no significant difference in the melting curves of PEU531 and PEU326 before and after UV irradiation, where only slightly increased crystallinity is observed. UV absorbers act as efficient barriers to prevent molecular chains from UV irradiation, and molecular chains remain their original state instead of moving around into crystal lattice. Therefore, slightly increased changes can be observed as to the samples added with UV absorber. In contrast, broken molecular chains of HDPE, PEA1010 and PEA168 occur during UV irradiation, and then the molecular chain easily moves for the lower inter-molecular entanglement. As a result, the molecular chain is easily discharged into the crystal lattice, and the crystallinity will be higher. On the other hand, the aging condition of a constant temperature of $65^{\circ} \mathrm{C}$ also gives a reasonable cause to the largely increased crystallinity because eventually the aging process is equivalent to a long anneal process. The movement of molecular chains leads to the increased crystallization.

The second melting curves of each sample are demonstrated in Figure 4b and the crystallinity is shown in Figure 4d. The melting point of HDPE and PEA1010 clearly shifted to the low temperature, while no changes can be observed in the melting points of PEA168, PEU531 and PEU326. After the heat history is eliminated, the generation of polar groups causes the improvement in the melting point of the matrix, yet the decrease in molecular weight resulting from molecular chain cleavage causes
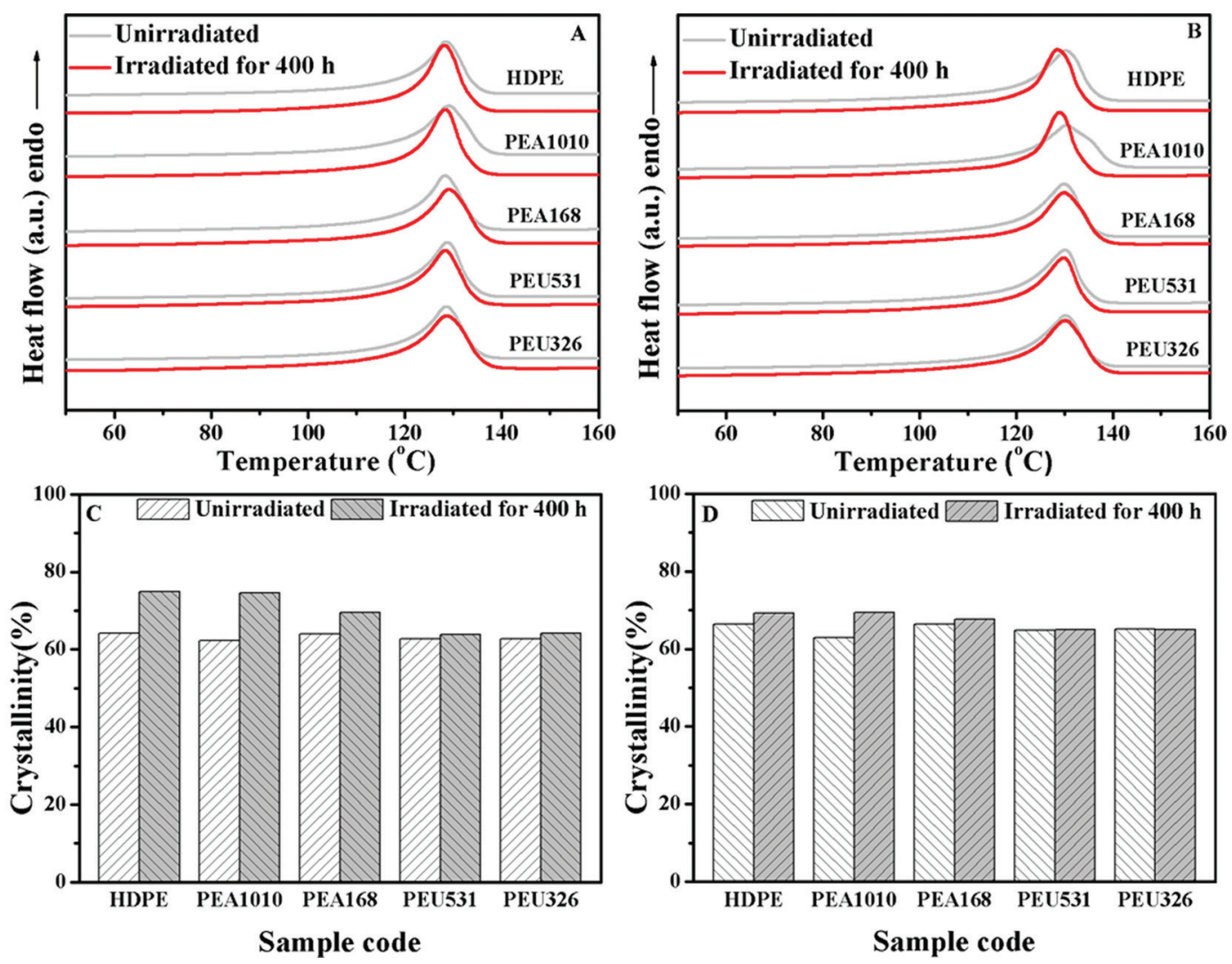

Figure 4: DSC curves and crystallinity of all samples before and after irradiation: (a) first melting curves; (b) second melting curves; (c) crystallinity on first melting curves; (d) crystallinity on second melting curves. 
the reduction in the melting point of the matrix. Since the produced carbonyl groups during UV irradiation were relatively few, their effect on the melting point can be ignored. However, the degree of molecular chain breakage of HDPE and PEA1010 maintains a high level, which consequently leads to the fact that the melting point is slightly decreased. With regard to PEA168, the degree of molecular chain breakage is alleviated for the decomposition of the macromolecular hydrogen peroxide. Meanwhile, the PEU series exhibit almost no molecular chain breaks for the presence of UV absorbers. Therefore, their melting points are almost unchanged. It can be seen from Figures $4 \mathrm{c}$ and $4 \mathrm{~d}$ that the crystallinity of the second heating curve of all unirradiated samples is higher than that obtained from the first heating curves. After irradiation for $400 \mathrm{~h}$, the crystallinity of HDPE, PEA1010 and PEA168 irradiation for $400 \mathrm{~h}$ are $69.2 \%, 69.4 \%$ and $65.8 \%$, respectively. The obtained crystallinity of the three samples decrease by $5.7 \%, 5.1 \%$ and $1.8 \%$, respectively, comparing with their first heating curve. On the contrary, the crystallinity of PEU is slightly higher than that of its first heating curve after irradiation for $400 \mathrm{~h}$. This is caused by the fact that the DSC system first heats up and then cools down to eliminate the thermal history, and eventually more time is allowed for the molecular chains with to circulate into the crystal lattice. At the same time, the effect of long-time annealing on the sample is also eliminated. Therefore, the crystallinity of HDPE, PEA1010 and PEA168 after irradiation for $400 \mathrm{~h}$ is lower than those of their heating curves without irradiation.
Since PEU531 and PEU326 have fewer molecular chain breaks, and as a result long-term annealing has little effect on them. Therefore, the crystallinity is slightly increased in comparison with the first heating curve after eliminating the history. Besides, at the second heating, the crystallinity of PEU531 and PEU326 before and after irradiation is almost unchanged.

The melted HDPE molecular chain is an amorphous random coiling macromolecule. Samples with good UV resistance, as well as unirradiated samples, have large molecular weight and long molecular chain length. However, samples with poor UV resistance and irradiated samples exhibit a small molecular weight and a short molecular chain for molecular chain breakage. Therefore, it is reasonable that the crystallization time of the same material largely differs from each other because of the difference in molecular chain length. As can be seen from the Scheme 1, the short molecular chains have been crystallized in a certain period of time under ideal conditions. The short molecular chain has a low degree of entanglement, is easy to discharge into the crystal lattice and takes a short time. During the cooling process, the long molecular chain is discharged into the crystal lattice at a slower rate than the short molecular chain due to the high degree of molecular chain entanglement and steric hindrance.

Table 1 is the results calculated by crystallinity curves of all samples before and after UV irradiation. The half crystallization time $\left(t_{1 / 2}\right)$, suggesting the time of half crystallinity, well reflects the crystallinity rate of

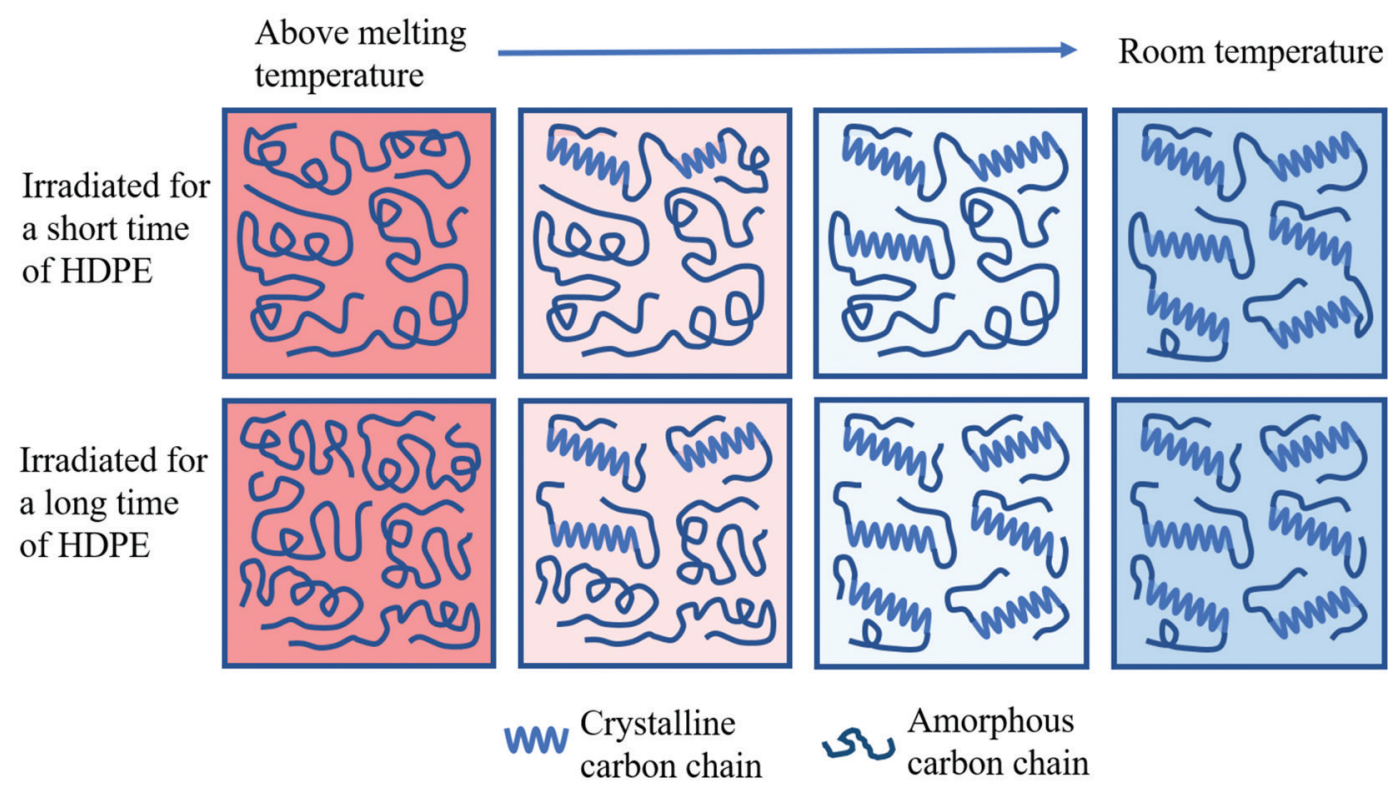

Scheme 1: Schematic mechanism of crystallization rate. 
the sample. The $t_{1 / 2}$ is calculated from the crystallinity starting temperature and the temperature at which the crystallinity is $50 \%$. The $t_{1 / 2}$ value of unirradiated HDPE is $28.8 \mathrm{~s}$, while it decreases to a value of $15.6 \mathrm{~s}$ after UV irradiation for $400 \mathrm{~h}$. This indicates that photo-oxidative

Table 1: Crystallization temperature and half crystallization time of all samples before and after UV irradiation.

\begin{tabular}{lccccc}
\hline $\begin{array}{l}\text { Samples } \\
\text { code }\end{array}$ & $\begin{array}{c}\text { Irradiation } \\
\text { time (h) }\end{array}$ & $T_{c s}\left({ }^{\circ} \mathrm{C}\right)$ & $T_{c 1 / 2}\left({ }^{\circ} \mathrm{C}\right)$ & $\Delta T_{c}\left({ }^{\circ} \mathrm{C}\right)$ & $t_{1 / 2}(\mathbf{s})$ \\
\hline HDPE & 0 & 119.8 & 117.4 & 2.4 & 28.8 \\
PEA1010 & 400 & 119.6 & 118.3 & 1.3 & 15.6 \\
& 0 & 119.6 & 116.6 & 3.0 & 36.0 \\
PEA168 & 0 & 119.6 & 118.3 & 1.3 & 15.6 \\
& 400 & 120.0 & 117.6 & 2.4 & 28.8 \\
PEU531 & 0 & 119.8 & 117.3 & 2.7 & 32.4 \\
& 400 & 120.1 & 117.7 & 2.3 & 27.6 \\
PEU326 & 0 & 119.7 & 117.1 & 2.6 & 31.2 \\
& 400 & 119.7 & 116.8 & 2.9 & 34.8 \\
\hline
\end{tabular}

$T_{c s}, T_{c 1 / 2}, \Delta T_{c}$ and $t_{1 / 2}$ represent crystallization starting temperature, half crystallization temperature, crystallization temperature range and half crystallization time, respectively. degradation of HDPE has occurred, and the crystallinity is increased. Because of the presence of the molecular chain breakage, the short molecular chain is more likely to be discharged into the crystal lattice. The $t_{1 / 2}$ value of unirradiated PEA1010 is significantly increased to $36.0 \mathrm{~s}$, indicating that the antioxidant 1010 hindered crystallinity of the polymer and lowered the crystallization rate. The antioxidant 1010 is consumed after UV irradiation for $400 \mathrm{~h}$, and the molecular chain fragmentation also increases the crystallization rate. The $t_{1 / 2}$ of PEA168, PEU531, PEU326 after UV irradiation is higher than those values before UV irradiation because of the formation of polar groups such as carbonyl. The existence of the polar groups destroys the regularity of HDPE matrix and produces steric hindrance effect, consequently making the molecule chains cannot be easily discharged into the crystal lattice.

\subsection{TGA analysis}

The thermal behavior of all samples was investigated by TGA under nitrogen atmospheres. The relative results of TGA test is illustrated in Figure 5, which shows the
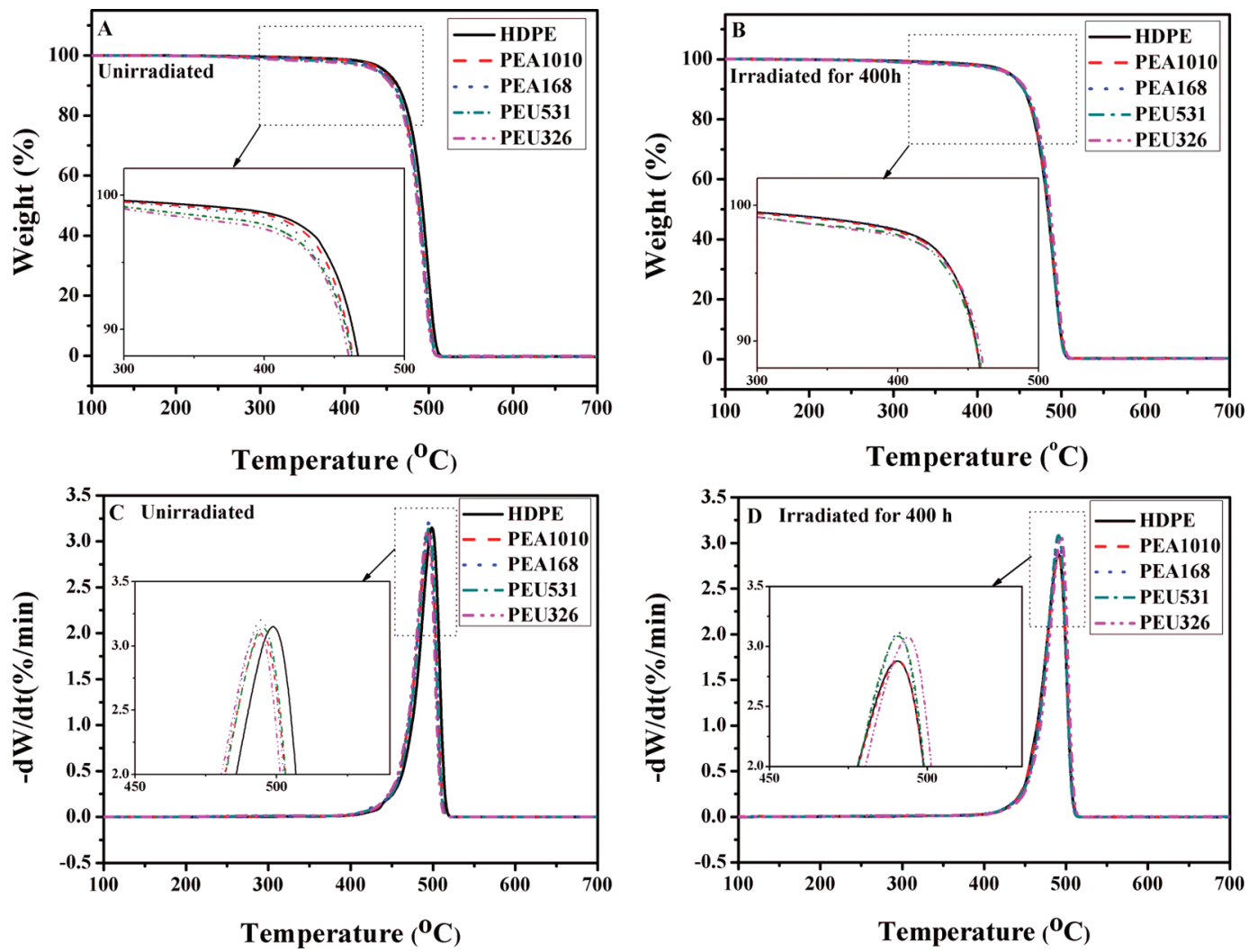

Figure 5: TGA thermograms (a) before irradiation (b) after UV irradiation and DTG curves (c) before irradiation (d) after UV irradiation of all samples. 
residue weight of samples at different temperatures. All samples degrade in one single step practically almost without any residue. Only one decomposition peak is obtained of all the samples, representing degradation of polyethylene at around the temperature of $430^{\circ} \mathrm{C}$ (27). The corresponding TGA data, including $\mathrm{T}_{5}, \mathrm{~T}_{25}, \mathrm{~T}_{50}$ and $\mathrm{T}_{\max }$, are summarized in Table 2. The phenomena leading to the weight loss due to thermal degradation can be further studied using the derivative weight loss (DTG) curves. In general, all samples have good thermal stability before and after UV irradiation, and there are no significant changes in thermal decomposition temperature. The addition of antioxidants and UV absorbers results in a slight decrease in the initial thermal decomposition temperature of HDPE. Compared with the unirradiated samples, only the $\mathrm{T}_{5}$ of HDPE and PEA1 decreases after UV irradiation, yet the changes of $\mathrm{T}_{5}$ of other samples can be almost ignored. The $\mathrm{T}_{\max }$ of PEU326 presents no change after irradiation, while other samples exhibit a slight decline in comparison with those samples without irradiation. In general, the irradiated sample also maintains the original excellent thermal stability.

\subsection{Surface topography}

Figure 6 shows the change in surface topography of all samples. The micrographs show that the nonaged samples are smooth, without any cracks. There are many spots on the surface of PEA168 and PEU326 that are evenly distributed, which are formed by the migration of Irgafos168 and UV326 to the surface of

Table 2: Degradation temperature of all samples before and after UV irradiation.

\begin{tabular}{lccccc}
\hline $\begin{array}{l}\text { Samples } \\
\text { code }\end{array}$ & $\begin{array}{c}\text { Irradiation } \\
\text { time }(\mathbf{h})\end{array}$ & $T_{5}\left({ }^{\circ} \mathrm{C}\right)$ & $T_{25}\left({ }^{\circ} \mathrm{C}\right)$ & $T_{50}\left({ }^{\circ} \mathrm{C}\right)$ & $T_{\max }\left({ }^{\circ} \mathrm{C}\right)$ \\
\hline HDPE & 0 & 447 & 479 & 492 & 499 \\
& 400 & 440 & 471 & 484 & 491 \\
PEA1010 & 0 & 444 & 476 & 488 & 495 \\
& 400 & 439 & 472 & 484 & 491 \\
PEA168 & 0 & 440 & 476 & 488 & 495 \\
& 400 & 439 & 473 & 485 & 491 \\
PEU531 & 0 & 438 & 476 & 489 & 495 \\
& 400 & 437 & 473 & 485 & 491 \\
PEU326 & 0 & 437 & 474 & 487 & 493 \\
& 400 & 439 & 475 & 487 & 494 \\
\hline
\end{tabular}

$T_{5}, T_{25}, T_{50}$ and $T_{\max }$ represent degradations of $10 \%, 25 \%, 50 \%$, and peak temperature of the derivative mass loss, respectively. sample, respectively. Since the antioxidant is quickly consumed, the spots on the surface of PEA168 disappear after irradiation for $200 \mathrm{~h}$. The spots on the surface of the sample become finer and more evenly dispersed after irradiation for $200 \mathrm{~h}$. As the aging time increases, the UV326 continues to migrate towards the surface of the sample. With the aging time of $400 \mathrm{~h}$, yellow crystals are observed to precipitate on the surface of the PEU326. After irradiated for $400 \mathrm{~h}$, it is found that the surfaces of PEA1010 and PEA168 present obvious microcracks like HDPE, but no obvious microcracks are observed on the surface of PEU531 and PEU326. Hence, UV absorbers perform better UV-resistance than antioxidant, which is in line with the FTIR and DSC analysis mentioned above.

Scheme 2 illustrates the UV resistance mechanism of the samples. As shown in the figure, the samples are all placed on the panel in the chamber, where the environment temperature maintains at $65^{\circ} \mathrm{C}$, and the ultraviolet is simulated with a xenon lamp to accelerate the aging process of the samples. Due to the addition of the UV absorber, the appearance of the PEU series is pale yellow. Although all the ultraviolet irradiates the matrix, the UV irradiation is largely absorbed by the UV absorbers, which preventing the damage to the matrix. However, as to other samples without the addition of UV absorbers, the UV irradiation directly enters the matrix. Under the continuous irradiation of UV light, the molecular chains of HDPE start to break from the amorphous region. After a long period of irradiation, the crystal region is also gradually broken. During the continuous irradiation process, the crystallinity significantly increases for the recrystallization of shorter molecular chains, but the results of the elongation at break and tensile strength decrease. The degradation mechanism of PEA1010 is similar to HDPE. Irganox1010 is quickly consumed by a large amount of free radicals. Since the UV resistance of the matrix material cannot be enhanced, the crystallinity in turns significantly increases. For the increase in crystallinity, the elongation at break is significantly reduced, and the tensile strength is also declined. The Irgafos 168 can decompose to form the carbonyl alkyl hydroperoxide, so that the degradation rate slows down. Also, the molecular chain breakage degree is significantly reduced, and the crystallinity is slightly increased. Consequently, the elongation at break decreased, but the tensile strength is not greatly declined. Since almost no molecular chains break and no change is observed in crystallization, both PEU531 and PEU326 display excellent UV resistance. 

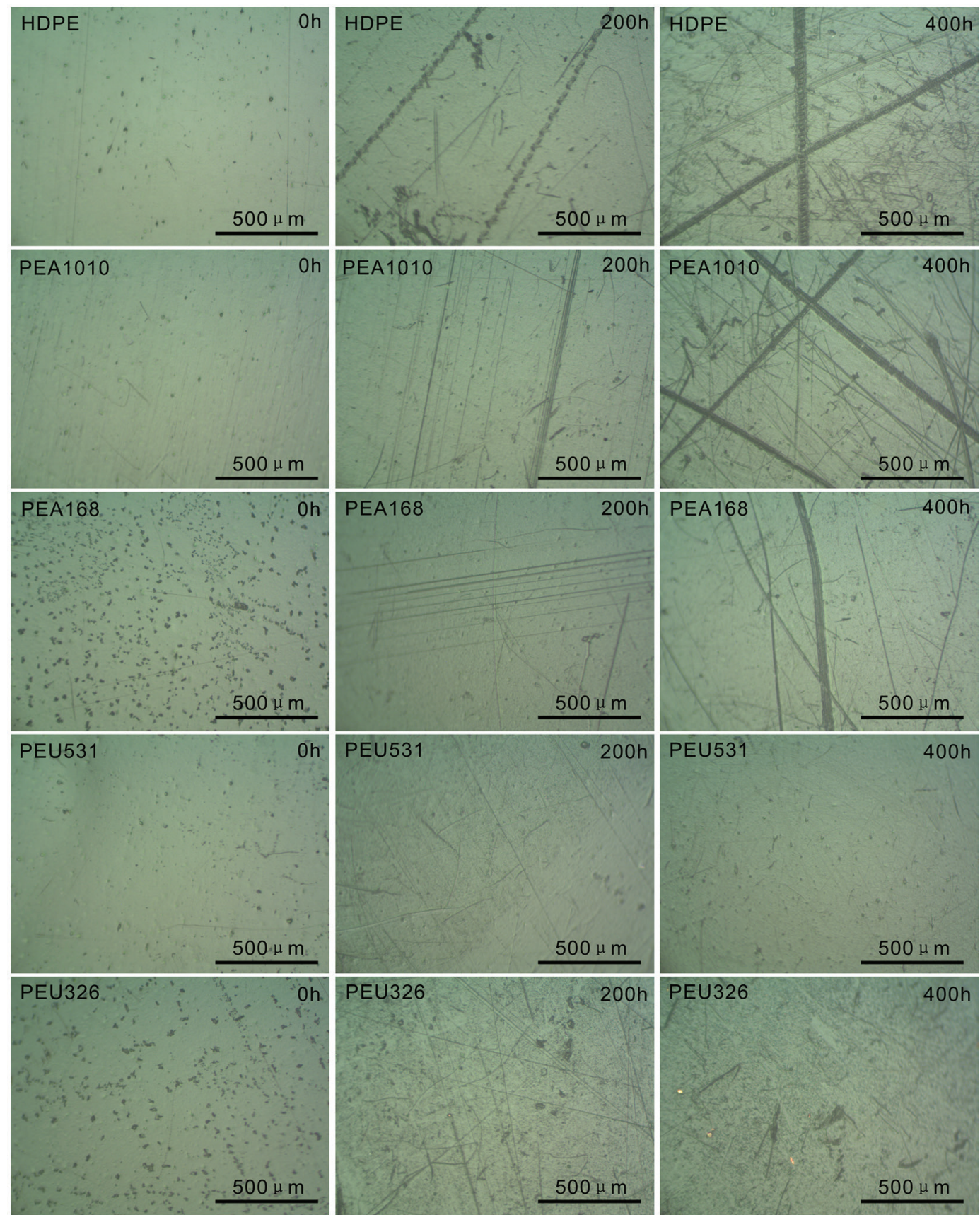

Figure 6: Optical micrograph of the surface of all samples at different times of UV irradiation.

\section{Conclusions}

In this study, antioxidant and UV absorber were applied to investigated their anti-aging performance based on the polymer matrix. Considering the UV-resistant mechanism, UV absorbers definitely exhibits much better UV resistance. The UV absorber efficiently absorbs UV light and reduces the generation of carbonyl groups. The addition of UV absorber can lead to the HDPE hardly undergo photo-oxidative degradation during irradiation, and the crystallinity was almost unchanged during the total UV irradiation process. However, the crystallization rate of the easy-aged samples was increased, as well as the crystallization, because short molecular chains were more likely to be discharged into the crystal lattices. The samples added with UV absorber also still maintain their mechanical properties at a high level even after irradiation for $600 \mathrm{~h}$. Yet the tensile 


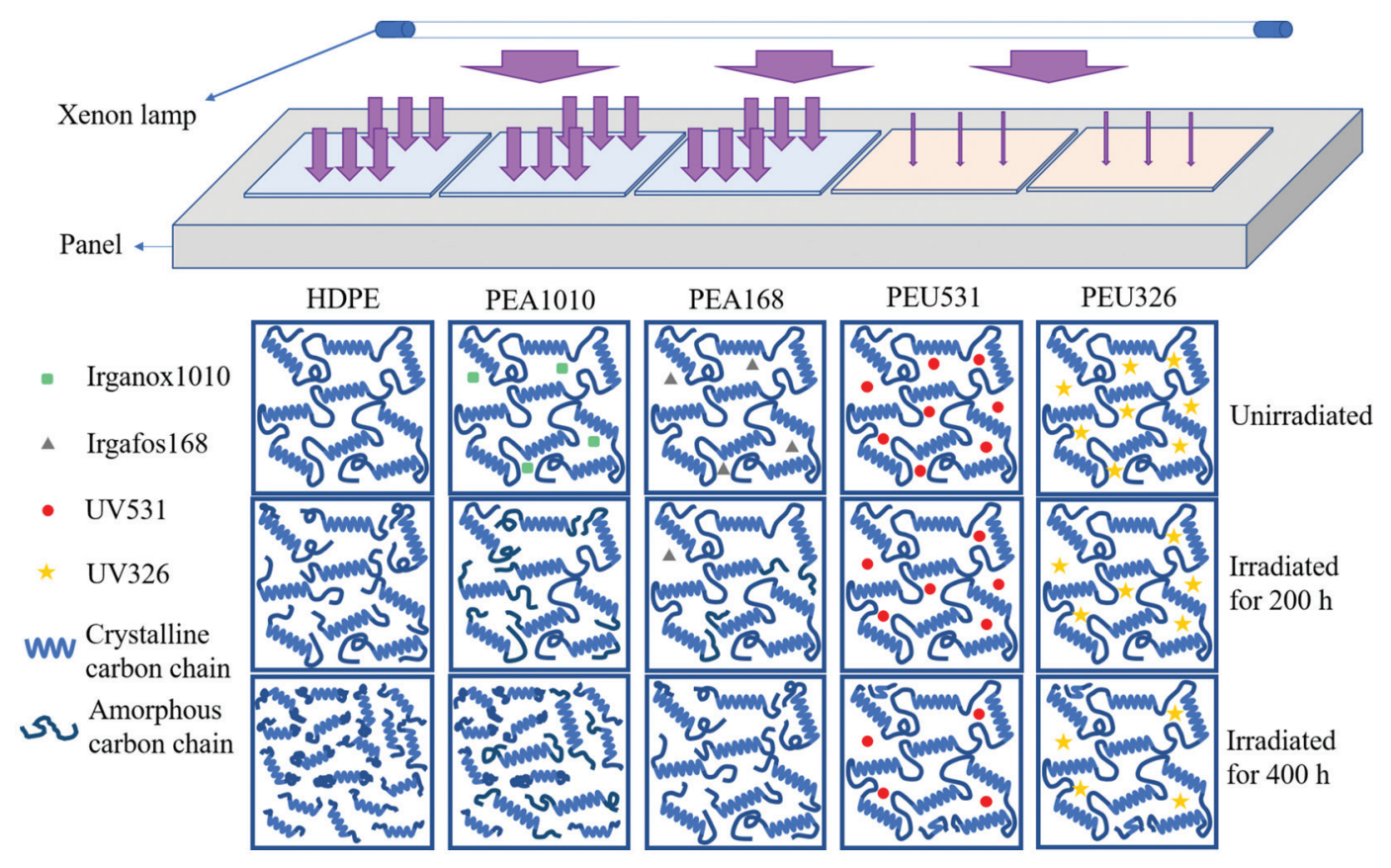

Scheme 2: UV resistance mechanism of all samples.

properties of the materials added with antioxidant significantly decreased after irradiation for only $200 \mathrm{~h}$, where the elongation at break decreased to almost $0 \%$ as the corresponding samples was irradiated for $200 \mathrm{~h}$. In summary, antioxidant performs slight improvement in the aging resistance of the HDPE, while the UV absorber exhibits excellent effect on the UV resistance of the material. Though excellent aging resistance for $600 \mathrm{~h}$ is obtained in this study, better weatherability is desirable through further research.

Acknowledgment: This work was supported by the Priority Academic Program Development of Jiangsu Higher Education Institution (PAPD).

\section{References}

1. Muniyandi S.K., Sohaili J., Hassan A., Converting non-metallic printed circuit boards waste into a value added product. J. Appl. Polym. Sci., 2016, 133, 43110.

2. Grigoriadou I., Pavlidou E., Paraskevopoulos K.M., Terzopoulou Z., Bikiaris D.N., Comparative study of the photochemical stability of HDPE/Ag composites. Polym. Degrad. Stabil., 2018, 153, 23-36.

3. Gomes V.N.C., Carvalho A.G., Furukava M., Medeiros E.S., Colombo C.R., Melo T.J.A., et al., Characterization of Wood Plastic Composite Based on HDPE and Cashew Nutshells Processed in a Thermokinetic Mixer. Polym. Compos., 2018, 39, 2662-2673.
4. Chipara M., Jones B., Chipara D.M., Li J.H., Lozano K., Valloppilly S., et al., On orientation memory in high density polyethylenecarbon nanofibers composites. e-Polymers, 2017, 17, 303-310.

5. Ahmad I., Khan M.I., Khan H., Ishaq M., Tariq R., Gul K., et al., Influence of metal-oxide-supported bentonites on the pyrolysis behavior of polypropylene and high-density polyethylene. J. Appl. Polym. Sci., 2015, 132, 41221.

6. Cuadri A.A., Martin-Alfonso J.E., The effect of thermal and thermooxidative degradation conditions on rheological, chemical and thermal properties of HDPE. Polym. Degrad. Stabil., 2017, 141, 11-18.

7. Majid F., Elghorba M., HDPE pipes failure analysis and damage modeling. Eng. Fail. Anal., 2017, 71, 157-166.

8. Bassiouny R., Ali M.R.O., Hassan M.K., An idea to enhance the thermal performance of HDPE pipes used for ground-source applications. Appl. Therm. Eng., 2016, 109, 15-21.

9. Su J., Zhang J., Preparation and properties of Barium titanate $\left(\mathrm{BaTiO}_{3}\right.$ ) reinforced high density polyethylene (HDPE) composites for electronic application. J. Mater. Sci.-Mater. El., 2016, 27, 4344-4350.

10. Adib A., Dominguez C., Garcia R.A., Garrido M.A., Rodriguez J., Influence of specimen geometry on the slow crack growth testing of HDPE for pipe applications. Polym. Test., 2015, 48, 104-110.

11. Tochacek J., Vratnickova Z., Polymer life-time prediction: The role of temperature in UV accelerated ageing of polypropylene and its copolymers. Polym. Test., 2014, 36, 82-87.

12. Briassoulis D., Babou E., Hiskakis M., Kyrikou I., Analysis of long-term degradation behavior of polyethylene mulching films with pro-oxidants under real cultivation and soil burial conditions. Environ. Sci. Pollut. Res. Int., 2015, 22, 2584-2598.

13. Habibi M., Najafi S.K., Ghasemi I., Rheological and mechanical properties of composites made from wood flour and recycled LDPE/HDPE blend. Iran. Polym. J., 2017, 26, 949-956. 
14. Rowe R.K., Ewais A.M.R., Ageing of exposed geomembranes at locations with different climatological conditions. Can. Geotech. J., 2015, 52, 326-343.

15. Ewais A.M.R., Rowe R.K., Rimal S., Sangam H.P., 17-year elevated temperature study of HDPE geomembrane longevity in air, water and leachate. Geosynth. Int., 2018, 25, 525-544.

16. Nguyen V.D., Hao J., Wang W., Ultraviolet Weathering Performance of High-Density Polyethylene/Wood-Flour Composites with a Basalt-Fiber-Included Shell. Polymers, 2018, 10, 831-843.

17. Grigoriadou I., Paraskevopoulos K.M., Karavasili M., Karagiannis G., Vasileiou A., Bikiaris D., HDPE/Cu-nanofiber nanocomposites with enhanced mechanical and UV stability properties. Compos Part B-Eng., 2013, 55, 407-420.

18. Grigoriadou I., Paraskevopoulos K.M., Chrissafis K., Pavlidou E., Stamkopoulos T.G., Bikiaris D., Effect of different nanoparticles on HDPE UV stability. Polym. Degrad. Stabil., 2011, 96, 151-163.

19. Park J.S., Kang P.H., Nho Y.C., Suh D.H., Effects of Thermal Ageing Treatment and Antioxidants on the Positive Temperature Coefficient Characteristics of Carbon Black/Polyethylene Conductive Composites. J. Appl. Polym. Sci., 2003, 8, 2316-2322.

20. Javadi Y., Hosseini M.S., Aghjeh M.K.R., The effect of carbon black and HALS hybrid systems on the UV stability of high-density polyethylene (HDPE). Iran. Polym. J., 2014, 23, 793-799.
21. Brandrup J., Immergut E.H., Grulke E.A. Polymer Handbook. Wiley, New York, 1999.

22. Jin J., Chen S.J., Zhang J., UV aging behaviour of ethylene-vinyl acetate copolymers (EVA) with different vinyl acetate contents. Polym. Degrad. Stabil., 2010, 95, 725-732.

23. Liu Z.Y., Chen S.J., Zhang J., Photodegradation of ethyleneoctene copolymers with different octene contents. Polym. Degrad. Stabil., 2011, 96, 1961-1972.

24. Khabbaz F., Albertsson A.C., Karlsson S., Trapping of volatile low molecular weight photoproducts in inert and enhanced degradable LDPE. Polym. Degrad. Stabil., 1998, 61, 329-342.

25. Wang S.C., Zhang J., Liu L., Yang F., Zhang Y.H., Evaluation of cooling property of high density polyethylene (HDPE)/titanium dioxide $\left(\mathrm{TiO}_{2}\right.$ ) composites after accelerated ultraviolet (UV) irradiation. Sol. Energ. Mat. Sol. C., 2015, 143, 120-127.

26. Huang Z.Y., Li Y.L., Ren X.C., Comparing cracking time and structure changes of different high-density polyethylenes during stress and photo-oxidative aging. J. Appl. Polym. Sci., 2014, 131, 40904.

27. Gulmine J.V., Akcelrud L., FTIR characterization of aged XLPE. Polym. Test., 2006, 25, 932-942.

28. Kord B., Ravanfar P., Ayrilmis N., Influence of Organically Modified Nanoclay on Thermal and Combustion Properties of Bagasse Reinforced HDPE Nanocomposites. J. Polym. Environ., 2017, 25, 1198-1207. 